USO DE LAS TIC EN EL PROCESO DE ENSEÑANZA-APRENDIZAJE EN LOS PROFESORES UNIVERSITARIOS.

\title{
USO DE LAS TIC EN EL PROCESO DE ENSEÑANZA - APRENDIZAJE EN LOS PROFESORES UNIVERSITARIOS
}

\section{USE OF THE ICT IN THE TEACHING - LEARNING PROCESS IN THE UNIVERSITY PROFESSORS}

\author{
Abraham Gordillo Mejía*, Cecilia García Muñoz Aparicio**
}

\begin{abstract}
*Doctor en Ciencias Económico Administrativas. Instituto Politécnico Nacional - UPIICSA. ORCID: http://orcid.org/0000-0001-5311-1810.

**Doctora en Ciencias Económico Administrativas. Universidad Juárez Autónoma de Tabasco. Email: flamingos1999@hotmail.com. ORCID: http://orcid.org/0000-0001-5316-8630.
\end{abstract}

Dirección para recibir correspondencia: abragorme@gmail.com 
USO DE LAS TIC EN EL PROCESO DE ENSEÑANZA-APRENDIZAJE EN LOS PROFESORES UNIVERSITARIOS.

\section{RESUMEN}

En la Unidad Profesional Interdisciplinaria de Ingeniería y Ciencias Sociales y Administrativas (UPIICSA), del Instituto Politécnico Nacional (IPN), se asume el reto de mantener la pertinencia en la sociedad, al ofrecer la carrera de Administración Industrial y que responda a las exigencias de la modernidad, pero sus estructuras y recursos humanos, pareciera que no lo hacen.

OBJETIVO: Analizar el uso de las TIC, en el proceso de enseñanza-aprendizaje de la Licenciatura en Administración Industrial desde la perspectiva de los docentes para elevar la calidad de la enseñanza.

MATERIAL Y MÉTODO: se utilizó un método no experimental, de tipo mixto cualitativo y cuantitativo ya que el fenómeno en estudio se observa y analiza tal como es en su contexto natural, además de realizar un análisis estadístico donde se aplicó una encuesta a una población seleccionada.

RESULTADOS: Se observó que existe una brecha, en cuanto a la pertinencia de la Licenciatura en Administración Industrial en el mercado, debido a que la carrera está desactualizada en relación con el progreso tecnológico, ya que su personal docente no está actualizado en nuevas modalidades y técnicas de enseñanza.

CONCLUSIONES: En la UPIICSA del IPN existe una percepción positiva en el uso de las TIC entre los docentes, los cuales están convencidos de su beneficio en clases para la transmisión de conocimientos.

PALABRAS CLAVE: Alumnos. Profesores. Proceso enseñanza-aprendizaje. TIC.

\section{ABSTRACT}

In the Interdisciplinary Professional Unit of Engineering and Social and Administrative Sciences (UPIICSA), of the Instituto Politécnico Nacional (IPN), the challenge of maintaining relevance in society and responding to the demands of modernity is assumed by offering the career of Industrial Administration, but it seems that its structures and human resources do not do it. 
USO DE LAS TIC EN EL PROCESO DE ENSEÑANZA-APRENDIZAJE EN LOS PROFESORES UNIVERSITARIOS.

OBJECTIVE: To analyze the use of ICT in the teaching - learning process of the Bachelor of Industrial Administration from the perspective of teachers to increase the quality of teaching.

MATERIAL AND METHOD: A non-experimental method of qualitative and quantitative mixed type was used since the phenomenon under study is observed and analyzed as it is in its natural context, in addition to carrying out a statistical analysis in which a survey was applied to a selected population.

RESULTS: It was observed that there is a gap, in regards to the relevance of the Bachelor of Industrial Administration in the market, due to the career is outdated in relation to technological progress, as its teaching staff is not updated in new modalities and teaching techniques.

CONCLUSIONS: In the UPIICSA of the IPN there is a positive perception in the use of ICT among teachers, who are convinced of their benefit in classes for the transmission of knowledge.

KEY WORDS: Students. Teachers. Teaching - learning process. ICT.

\section{INTRODUCCIÓN}

En los nuevos modelos educativos como el constructivismo, donde los profesores deben jugar un rol más participativo, como facilitadores y tutores en la docencia, se ha percibido de manera generalizada que su función la realizan de forma tradicional en el modelo presencial conductista, y aunque existen escenarios en que los profesores utilizan las Tecnologías de la Información y la Comunicación (TIC), las cuales no forman parte vital del proceso de enseñanza- aprendizaje, sino como un apoyo, ellos utilizan el correo electrónico, los chat, las redes sociales, para efectos de subir apuntes en la Web, crear equipos virtuales de colaboración, simuladores digitales, y evaluaciones en línea, almacenamiento y distribución de materiales en la nube, entre otras actividades, pero a discreción del profesor, sin ser obligatorio.

Estas situaciones observadas y comentadas por el Jefe de la carrera de la Licenciatura en Administración Industrial de la Unidad Profesional Interdisciplinaria de Ingeniería y Ciencias 
USO DE LAS TIC EN EL PROCESO DE ENSEÑANZA-APRENDIZAJE EN LOS PROFESORES UNIVERSITARIOS.

Sociales y Administrativas (UPIICSA) del Instituto Politécnico Nacional (IPN) (2017), proporcionaron la pauta para realizar un estudio que permita determinar la percepción que tienen los profesores de dicha carrera, sobre el uso que le dan a las TIC en el proceso de enseñanza-aprendizaje; adicional a la opinión del Jefe de Carrera, se encontró que en la evaluación externa, los empleadores solicitan egresados en dominio de las TIC; en estudios comparativos de curriculas con otras universidades que ofrecen estudios de Administración, estas incluyen el uso de las TIC en sus procesos de enseñanza-aprendizaje, además de contar con modalidades mixtas y a distancia; como recomendación del Consejo de Acreditación en la Enseñanza de la Contaduría y Administración (CACECA) se requiere implantar programas de actualización de competencias ligadas al uso de las TIC. En la evaluación interna, se referenció lo declarado en el Modelo Educativo del IPN, donde solicita que todos los programas de estudio del instituto estén centrados en el aprendizaje y que además de manera permanente, diseñen y establezcan metodologías de la enseñanza que otorguen prioridad a la innovación, a la creatividad y al uso intensivo de las TIC (IPN, 2004).

Por tanto, requiere que los profesores y actores en el proceso de enseñanza-aprendizaje, construyan espacios para la adquisición del conocimiento, con académicos que aprendan enseñando y que incorporen en su práctica docente el uso de las TIC de forma intensiva, para lograr incrementar la calidad educativa de las carreras que se ofrecen y en particular en dicha licenciatura.

\section{DESARROLLO}

\section{Calidad de la enseñanza}

Márquez, (2004, citando a Muñoz et al. 1998), considera que la calidad educativa es un concepto normativo integrado por las dimensiones filosófica, pedagógica, económica, cultural y social. Dichas dimensiones generan un sistema de calidad, en lo filosófico se considera que sus contenidos son de interés a la sociedad y dan respuesta a ciertas necesidades; en lo pedagógico se pretende cubrir de manera eficaz los objetivos propuestos en los planes y programas; en lo económico, que los recursos asignados sean utilizados eficientemente; en lo cultural, que los contenidos y métodos de la enseñanza resulten pertinentes en el proceso de aprendizaje; y en lo social, la calidad se logra cuando están equitativa o igualitariamente distribuidas las oportunidades de acceso a la sociedad. 
USO DE LAS TIC EN EL PROCESO DE ENSEÑANZA-APRENDIZAJE EN LOS PROFESORES UNIVERSITARIOS.

En México, una institución que evalúa los elementos de calidad en las universidades, es la ANUIES (Asociación Nacional de Universidades e Instituciones de Educación Superior), la cual acuñó el término Aseguramiento de la Calidad. Dicha institución y sus normas, interpretadas por Barroso (2010), comenta que hay que tomar en cuenta tres criterios: Eficiencia de los procesos; Eficacia de los resultados; y Congruencia y relevancia de dichos procesos y sus resultados con las expectativas y demandas sociales; estos requisitos las Instituciones de Educación Superior (IES), los adoptan y adaptan a sus procesos internos, tal es el caso de la afirmación de Doger (1994) que en un documento sobre las medidas para asegurar la calidad, afirma que la calidad en educación superior se manifiesta a través de los procesos de generación, transmisión y preservación del conocimiento y al impacto que tengan los procesos de enseñanza a cubrir las necesidades y problemas de la sociedad.

Rodríguez (2014), afirma que pueden ser, áulico (real), y virtual; en el primero, el proceso de enseñanza-aprendizaje se desarrolla en el aula (salón, laboratorio, sala, biblioteca), escenarios cuya ventaja es la vivencia directa en una relación de aprendizaje, comprobando grado de conocimientos y habilidades adquiridos, por lo que los niveles de calidad son más fáciles de reconocerlos, medirlos y comprobarlos de manera directa por el docente.

La UPIICSA del IPN se encuentra en esta transición, de incorporar de forma total el uso de las TIC, creando esos ambiente y escenarios virtuales que apoyen el aprendizaje en la modalidad presencial, donde el profesor tiene que desarrollar todos los elementos de un ambiente de aprendizaje, ya que él tiene que jugar todos los roles: diseña, escribe, plantea, determina medios, formatos y tiempos, y de acuerdo con García (2017) el rendimiento de los estudiantes estriba a partir de los diseños pedagógicos, así como los recursos seleccionados para el aprendizaje, debido a que con diseños rigurosos, en cualquier tipo de formato ya sea virtual, presencial o a distancia no existe diferencia en los resultados; por ello, en este siglo no se pone en duda la eficiencia de los proyectos educativos no presenciales.

\section{Diseño de la investigación}

Esta investigación se realizó con base en las necesidades internas de la UPIICSA, referenciadas por la Jefatura de carrera de Licenciatura en Administración Industrial, que además de constituirse como una investigación documental, realizó un estudio de campo, 
USO DE LAS TIC EN EL PROCESO DE ENSEÑANZA-APRENDIZAJE EN LOS PROFESORES UNIVERSITARIOS.

donde la pregunta principal fue: ¿qué uso de las TIC, hacen los profesores y alumnos de dicha licenciatura?

Es una investigación no experimental, ya que el fenómeno en estudio se observa y analiza tal como es en su contexto natural, y ninguna de las variables de estudio se puede modificar; además de ser mixta: cualitativa, por ubicarse dentro de las ciencias sociales, en donde no hay paradigmas referenciales, y cuantitativa, ya que se realizó un análisis estadístico numérico.

Para este estudio se definieron las variables independientes y dependientes, el diseño de un cuestionario, el cálculo de una muestra, cuyo resultado fue de 138 profesores, de las 6 academias del área de ciencias sociales. El diseño del cuestionario se adaptó a tres variables o dimensiones en consideración: Actitud (de los profesores hacia el uso de las TIC), Conocimiento (nivel adquirido de formación en el uso de las TIC) y Nivel de uso (grado de uso de las TIC en su actividad docente); el cuestionario se compuso de 34 ítems, con respuestas múltiples de acuerdo al modelo de Hernández, Maquilón, Monroy e Izquierdo (2010), con una escala Likert de cinco respuestas posibles.

Se realizó una validación del constructo, aplicando un pilotaje a 50 profesores, tratando con ello de obtener resultados exactos que llevarán a resultados válidos (Colás y Buendia, 1998), además se sometió a la prueba de validez atendiendo a las sugerencias de larossi (2006), y de confiabilidad aplicando el test Alfa de Cronbach, el cual es recomendado para investigaciones que utilicen cuestionarios con rangos de respuesta para cada ítem (McMillan y Schumacher, 2011). Los resultados de confiabilidad fueron cuatro: el primero de fue el resultado global con un alfa de Cronbach de .93; en los ítems de Actitud ante el uso de TIC, de .93, en la dimensión Conocimiento de TIC, de .89, y en uso de TIC fue de .86, que de acuerdo a Merino y Lautenschlager (2003), en cuanto más se acerque el valor de Alfa a 1, mayor es la consistencia interna del constructo. En cuanto a los resultados, se inició con un análisis de los datos genéricos de identificación de profesores, considerando el total de profesores encuestados/academia, su formación, edad, antigüedad del profesorado en su labor docente y antigüedad del profesorado en su labor no docente.

Uno de los análisis realizados fue el de promedios y desviaciones estándar de las respuestas de los profesores en los ítems planteados y poder con ello realizar una comparación de comportamiento por cada dimensión (ver figura 1). 
USO DE LAS TIC EN EL PROCESO DE ENSEÑANZA-APRENDIZAJE EN LOS PROFESORES UNIVERSITARIOS.

Los principales resultados obtenidos se presentan en la tabla 1 y en la figura 1, con análisis reflexivo de la información obtenida.

\section{Tabla 1}

Resumen de resultados de datos genéricos de los profesores

\begin{tabular}{|c|c|}
\hline Factor & Comentario \\
\hline Formación & $\begin{array}{l}\text { La plantilla docente se integra por un } 45.65 \% \text { de Licenciados, un } 47.10 \% \text { de } \\
\text { Maestros en Ciencias y un } 7.25 \% \text { de Doctores, situación normal en la UPIICSA, } \\
\text { donde existen estrategias para que los profesores se especialicen y eleven su } \\
\text { formación. }\end{array}$ \\
\hline Edad profesores & $\begin{array}{l}\text { Un } 63.05 \% \text { de profesores son mayores de } 51 \text { años, con lo cual se observa una } \\
\text { plantilla "mayor", con ventajas de experiencia y conocimientos en sus áreas de } \\
\text { dominio, pero con desventajas inherentes a la edad. }\end{array}$ \\
\hline Academia & $\begin{array}{l}\text { En la encuesta participaron profesores de todas las academias que conforman el } \\
\text { departamento de Ciencias Sociales, cubriendo una muestra de } 138 \text { profesores, } \\
\text { escogida totalmente de forma aleatoria. }\end{array}$ \\
\hline $\begin{array}{l}\text { Antigüedad como } \\
\text { profesor }\end{array}$ & $\begin{array}{l}\text { En este factor se encontró un dato relevante: un } 55.07 \% \text { de la muestra tiene más } \\
\text { de } 20 \text { años en la docencia, lo que constituye una ventaja debido al conocimiento y } \\
\text { experiencia adquirido en dicho periodo, sin embargo existen profesores, con menos } \\
\text { de } 5 \text { años de experiencia, lo cual puede percibirse como una debilidad (25\%). }\end{array}$ \\
\hline $\begin{array}{l}\text { Antigüedad no } \\
\text { docente }\end{array}$ & $\begin{array}{l}\text { Un } 51.45 \% \text { de profesores tiene más de } 20 \text { años en la vida profesional, lo que } \\
\text { combinado con la experiencia docente, forma una combinación académica-práctica } \\
\text { en los procesos de enseñanza. }\end{array}$ \\
\hline
\end{tabular}

Fuente: Elaboración propia.

La tabla 1, permite describir de manera sintética algunas características relevantes positivas como experiencia de la plantilla docente, más de un $40 \%$ cuentan con estudios de posgrado en su área disciplinar y pedagógica así también se observan algunas negativas como la edad (51 años en adelante), falta de experiencia (los que tienen menos de 5 años). 
USO DE LAS TIC EN EL PROCESO DE ENSEÑANZA-APRENDIZAJE EN LOS PROFESORES UNIVERSITARIOS.

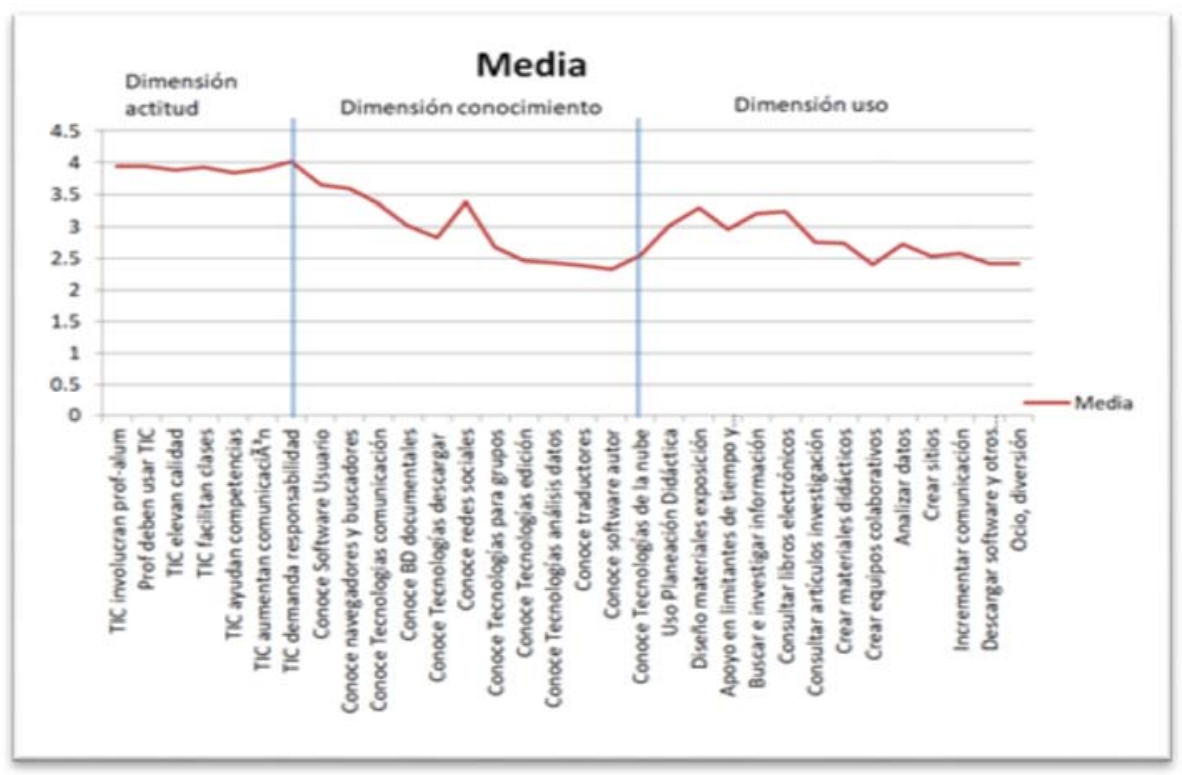

Figura 1. Comparativo de los valores medios obtenidos de las respuestas dada por los profesores en cada ítem/dimensión.

Fuente: Elaboración propia.

En la figura 1, permite realizar deducciones objetivas sobre la percepción que tienen los profesores respecto de las TIC; en el análisis de promedios realizado sobre la escala Lickert, se aprecia que los profesores poseen una buena percepción personal en la dimensión de actitud, para que se usen las TIC en los procesos de enseñanza-aprendizaje.

En relación a la dimensión del conocimiento sobre las TIC, ocurrió un comportamiento "extraño", calificándolo de esta forma, porque ante la actitud positiva para el uso de las TIC de la dimensión anterior, los profesores solo conocen algunos tipos de TIC, las de uso común de todo usuario de la web y del internet, como son los navegadores y buscadores, el correo electrónico y algunas redes sociales, entre otras básicas; el comportamiento respecto de la media de uso, disminuye drásticamente hasta el nivel de 2.5 , que corresponde a opciones de desconocimiento de ellas.

En la dimensión uso de las TIC, se encontró el mismo comportamiento de la dimensión anterior, lo que asegura la consistencia en las respuestas; el uso de las TIC como apoyo a las actividades del proceso, se limitó a nueve de ellas muy representativas (ver figura 1), en el tercer cuadrante de la derecha, para referenciarlas y conformarlas se tomó el criterio de Cooper, (1999) y Richards y Rodgers (1992). 
USO DE LAS TIC EN EL PROCESO DE ENSEÑANZA-APRENDIZAJE EN LOS PROFESORES UNIVERSITARIOS.

El promedio de respuestas osciló entre 2.5 y 3.5; es decir, los profesores las utilizan ocasionalmente en las actividades de enseñanza predominando aquellas relacionadas con la planeación didáctica, el diseño de materiales y la búsqueda de información, no trascendiendo a otras actividades relevantes del proceso. De la información obtenida y reflexionada, se realizaron algunos trabajos adicionales de análisis de datos y al tratar de precisar más conocimiento sobre este estudio, fue necesario realizar el cruce de diferentes variables, buscando y verificando que realmente exista una relación entre ellas, algunos cruces relevantes, fueron:

\section{1) Actitud hacia las TICledad profesores}

En la tabla 2, se observa que de toda la muestra un $38.41 \%$ de profesores, están totalmente de acuerdo en usar las TIC, con profesores entre 36 y 50 años, que son los más dispuestos a utilizarlas; pero los mayores de 50 años, representando un 20\%, son los más renuentes al uso de las TIC, pero son importantes por su experiencia y antigüedad.

Tabla 2

Tabla cruzada entre edad de los profesores vs ítems si los profesores deben usar las TIC

\begin{tabular}{llllll}
\hline Profesores que deben usar TIC & $\begin{array}{c}\text { de } 25 \text { a } 35 \\
\text { años }\end{array}$ & $\begin{array}{c}\text { de } 36 \text { a } 50 \\
\text { años }\end{array}$ & $\begin{array}{c}\text { de } 51 \text { a } 65 \\
\text { años }\end{array}$ & $\begin{array}{l}\text { de } 66 \text { en } \\
\text { adelante }\end{array}$ & $\begin{array}{l}\text { Total } \\
\text { Nada de acuerdo }\end{array}$ \\
& 0 & 0 & 0 & 9 & 9 \\
& $0.00 \%$ & $0.00 \%$ & $0.00 \%$ & $22.50 \%$ & $6.52 \%$ \\
& $0.00 \%$ & $0.00 \%$ & $0.00 \%$ & $6.52 \%$ & $6.52 \%$ \\
En desacuerdo & 0 & 0 & 0 & 3 & 3 \\
& $0.00 \%$ & $0.00 \%$ & $0.00 \%$ & $7.50 \%$ & $2.17 \%$ \\
Indiferente & $0.00 \%$ & $0.00 \%$ & $0.00 \%$ & $2.17 \%$ & $2.17 \%$ \\
& 3 & 7 & 6 & 12 & 28 \\
De acuerdo & $30.00 \%$ & $17.07 \%$ & $12.77 \%$ & $30.00 \%$ & $20.29 \%$ \\
& $2.17 \%$ & $5.07 \%$ & $4.35 \%$ & $8.70 \%$ & $20.29 \%$ \\
Totalmente de acuerdo & 3 & 14 & 20 & 8 & 45 \\
& $30.00 \%$ & $34.15 \%$ & $42.55 \%$ & $20.00 \%$ & $32.61 \%$ \\
& $2.17 \%$ & $10.14 \%$ & $14.49 \%$ & $5.80 \%$ & $32.61 \%$ \\
\hline
\end{tabular}

Fuente: Elaboración propia. 
USO DE LAS TIC EN EL PROCESO DE ENSEÑANZA-APRENDIZAJE EN LOS PROFESORES UNIVERSITARIOS.

\section{2) Edad profesores/ si las TIC facilitan las clases}

En la tabla 3, se analiza que un $34.78 \%$ está totalmente de acuerdo y un $36.23 \%$ de acuerdo, sumando respuestas en un $71.01 \%$, considerando a profesores entre 25 y 51 años.

\section{Tabla 3}

Tabla cruzada de edad vs las TIC que facilitan las clases

\begin{tabular}{|c|c|c|c|c|c|}
\hline TIC que facilitan clases & $\begin{array}{c}\text { de } 25 \text { a } 35 \\
\text { años }\end{array}$ & $\begin{array}{c}\text { de } 36 \text { a } 50 \\
\text { años }\end{array}$ & $\begin{array}{c}\text { de } 51 \text { a } 65 \\
\text { años }\end{array}$ & $\begin{array}{l}\text { de } 66 \text { en } \\
\text { adelante }\end{array}$ & Total \\
\hline Nada de acuerdo & $\begin{array}{c}0 \\
0.00 \%\end{array}$ & $\begin{array}{c}0 \\
0.00 \%\end{array}$ & $\begin{array}{c}0 \\
0.00 \%\end{array}$ & $\begin{array}{c}8 \\
20.00 \%\end{array}$ & $\begin{array}{c}8 \\
5.80 \%\end{array}$ \\
\hline En desacuerdo & $\begin{array}{c}0 \\
0.00 \%\end{array}$ & $\begin{array}{c}0 \\
0.00 \%\end{array}$ & $\begin{array}{c}0 \\
0.00 \%\end{array}$ & $\begin{array}{c}3 \\
7.50 \%\end{array}$ & $\begin{array}{c}3 \\
2.17 \%\end{array}$ \\
\hline Indiferente & $\begin{array}{c}1 \\
10.00 \%\end{array}$ & $\begin{array}{c}7 \\
17.07 \%\end{array}$ & $\begin{array}{c}9 \\
19.15 \%\end{array}$ & $\begin{array}{c}12 \\
30.00 \%\end{array}$ & $\begin{array}{c}29 \\
21.01 \%\end{array}$ \\
\hline De acuerdo & $\begin{array}{c}3 \\
30.00 \%\end{array}$ & $\begin{array}{c}17 \\
41.46 \%\end{array}$ & $\begin{array}{c}19 \\
40.43 \%\end{array}$ & $\begin{array}{c}11 \\
27.50 \%\end{array}$ & $\begin{array}{c}50 \\
36.23 \%\end{array}$ \\
\hline Totalmente de acuerdo & $\begin{array}{c}6 \\
60.00 \%\end{array}$ & $\begin{array}{c}17 \\
41.46 \%\end{array}$ & $\begin{array}{c}19 \\
40.43 \%\end{array}$ & $\begin{array}{c}6 \\
15.00 \%\end{array}$ & $\begin{array}{c}48 \\
34.78 \%\end{array}$ \\
\hline Total & $\begin{array}{c}10 \\
100.00 \%\end{array}$ & $\begin{array}{c}41 \\
100.00 \%\end{array}$ & $\begin{array}{c}47 \\
100.00 \%\end{array}$ & $\begin{array}{c}40 \\
100.00 \%\end{array}$ & $\begin{array}{c}138 \\
100.00 \%\end{array}$ \\
\hline
\end{tabular}

Fuente: Elaboración propia.

\section{3) Actitud hacia las TIC/formación profesores}

En la tabla 4, se encontró que los profesores con formación de maestría, son los que apoyan el uso de las TIC, con un 53.85\% totalmente de acuerdo y un $26.15 \%$ de acuerdo; en segundo lugar, están los profesores con nivel de licenciatura y en tercero, los profesores con nivel de doctorado, lo cual se considera un hallazgo, ya que son los que tienen el grado más alto de preparación. 
USO DE LAS TIC EN EL PROCESO DE ENSEÑANZA-APRENDIZAJE EN LOS PROFESORES UNIVERSITARIOS.

\section{Tabla 4}

Tabla cruzada entre la formación de profesores vs uso de las TIC por el profesor

\begin{tabular}{lcccc}
\hline Profesor deben usar TIC & Licenciatura & Maestría & Doctorado & Total \\
\hline Nada de acuerdo & 8 & 1 & 0 & 9 \\
& $12.70 \%$ & $1.54 \%$ & $0.00 \%$ & $6.52 \%$ \\
En desacuerdo & 3 & 0 & 0 & 3 \\
& $4.76 \%$ & $0.00 \%$ & $0.00 \%$ & $2.17 \%$ \\
Indiferente & 13 & 12 & 3 & 28 \\
& $20.63 \%$ & $18.46 \%$ & $30.00 \%$ & $20.29 \%$ \\
De acuerdo & 25 & 17 & 3 & 45 \\
& $39.68 \%$ & $26.15 \%$ & $30.00 \%$ & $32.61 \%$ \\
Totalmente de acuerdo & 14 & 35 & 4 & 53 \\
& $22.22 \%$ & $53.85 \%$ & $40.00 \%$ & $38.41 \%$ \\
Total & 63 & 65 & 10 & 138 \\
& $100.00 \%$ & $100.00 \%$ & $100.00 \%$ & $100.00 \%$ \\
\hline
\end{tabular}

Fuente: Elaboración de propia.

\section{4) Edad profesores/si las TIC aumentan la calidad de la enseñanza}

La tabla 5 presenta que un 33\% de la muestra está totalmente de acuerdo con la calidad de la enseñanza y otro $36.23 \%$, también, sumando un $66.23 \%$ de respuestas positiva.

Tabla 5

Tabla cruzada entre la variable edad vs las TIC elevan la calidad de la enseñanza

\begin{tabular}{lccccc}
\hline TIC elevan calidad & $\begin{array}{c}\text { de } 25 \text { a } 35 \\
\text { años }\end{array}$ & $\begin{array}{c}\text { de } 36 \text { a } 50 \\
\text { años }\end{array}$ & $\begin{array}{c}\text { de } 51 \text { a } 65 \\
\text { años }\end{array}$ & $\begin{array}{c}\text { de } 66 \text { en } \\
\text { adelante }\end{array}$ & Total \\
\hline Nada de acuerdo & 0 & 0 & 0 & 10 & 10 \\
Indiferente & $0.00 \%$ & $0.00 \%$ & $0.00 \%$ & $25.00 \%$ & $7.25 \%$ \\
& 3 & 7 & 8 & 14 & 32 \\
De acuerdo & $30.00 \%$ & $17.07 \%$ & $17.02 \%$ & $35.00 \%$ & $23.19 \%$ \\
& 4 & 15 & 21 & 10 & 50 \\
Totalmente de & $40.00 \%$ & $36.59 \%$ & $44.68 \%$ & $25.00 \%$ & $36.23 \%$ \\
acuerdo & 3 & 19 & 18 & 6 & 46 \\
& $30.00 \%$ & $46.34 \%$ & $38.30 \%$ & $15.00 \%$ & $33.33 \%$ \\
Total & 10 & 41 & 47 & 40 & 138 \\
& $100.00 \%$ & $100.00 \%$ & $100.00 \%$ & $100.00 \%$ & $100.00 \%$ \\
\hline
\end{tabular}

Fuente: Elaboración propia. 

UNIVERSITARIOS.

\section{CONCLUSIONES}

Para Fernández (2017), la educación superior es un bien estratégico para las naciones, conformando un lazo entre la generación de recurso humano capacitado, así como la producción y difusión de conocimientos que favorecen sociedades y economías competitivas, por ello, las TIC en su contexto teórico prometen beneficios en la labor de enseñanza, ya que apoyan la labor docente y normalmente son consideradas como competencias transversales, donde es evidente su uso por los profesores; sin embargo, esto no sucede así, siendo una realidad a enfrentar. En la UPIICSA del IPN, existe una percepción positiva entre los docentes para su uso, ya que reconocen sus beneficios en el desarrollo de las clases, en la transmisión de la información y conocimiento y sin duda ayudan a elevar la calidad educativa, al mismo tiempo existe una aparente contradicción de que los docentes conocen pocas a las TIC y por consecuencia no las usan.

En ese sentido, se vuelve evidente el reto para la gestión de la actividad académica, que de acuerdo con UNESCO (2009): la educación superior debe incrementar la formación docente con currículos que provean los conocimientos y las herramientas necesarias para el siglo XXI. Esto requerirá nuevos abordajes, que incluyan la Educación Abierta y a Distancia (EAD) e incorporen nuevas TIC.

Además, para las autoridades y profesores de la UPIICSA es un desafío, debido a que en los resultados se puede percibir un choque generacional entre una plantilla de docentes con mucha experiencia docente y laboral, pero cuya práctica docente es inamovible, basada en modelos tradicionales de enseñanza y evaluación, donde semestre a semestre replican sus clases, ponen a exponer a los alumnos sin retroalimentación, y su innovación y creatividad en el diseño de nuevas formas materiales y ambientes eleven la transmisión del conocimiento a otros niveles de calidad. Siendo la problemática inicial hablar de la posibilidad de introducir las TIC en la práctica docente, donde lo digital y virtual tienen que convivir en ambientes presenciales enriquecidos formalmente con el uso de las TIC, siendo esta parte de los planes y programas de estudio.

Por tanto, UPIICSA se debe añadir a la lista de instituciones que ya han enfrentado estos retos, como han sido las universidades de Jaén, (Rivera, 2008); la de Andalucía, la de Málaga, Sevilla y Huelva, según las investigaciones de Barroso (2010), sin olvidarse de la Pontificia Universidad 
USO DE LAS TIC EN EL PROCESO DE ENSEÑANZA-APRENDIZAJE EN LOS PROFESORES UNIVERSITARIOS.

de República Dominicana, (Cabero, Llorente y Puentes, 2010), y de la Complutense de Madrid, (Fernández et al, 2010), son muestras de los intentos a nivel mundial y donde hay muchos éxitos en proyectos de esta naturaleza, pero también hay fracasos, lo que deja ver la dificultad de este tipo de problemas en el ámbito educativo.

Finalmente, se observa que la pertinencia de la Licenciatura en Administración Industrial en el mercado y en la sociedad debe reposicionarse con acciones, introduciendo a las TIC en sus estrategias educativas y en los procesos de enseñanza-aprendizaje y subir con ello la demanda detectada por las autoridades correspondientes.

\section{REFERENCIAS BIBLIOGRÁFICAS}

Barroso, C. (2010). Acercamiento a las nuevas modalidades educativas en el IPN. Innovación Educativa, 6(30), 5-16. Recuperado de http://www.redalyc.org/pdf/1794/179420843002.pdf

Barroso, J. y Cabero, J. (2010). Valoraciones de los alumnos sobre el e-learning en las universidades andaluzas. Edutec. Revista Electrónica de Tecnología Educativa, (31). Recuperado de https://www.researchgate.net/publication/236856317_Usos_del_elearning_en_las_Universidades_Andaluzas_Estado_de_la_situacion_y_analisis_de_buen as_practicas.

Cabero, J., Llorente, C. y Puentes, A. (2010). La satisfacción en los estudiantes en red en la formación semipresencial. Comunicar, 18(35), 149-157.

Colás, P. y Buendía, L. (1998). Investigación educativa. España: Alfar.

Cooper, J. (1999). Estrategias de enseñanza: guía para una mejor enseñanza. México: Limusa. Doger, J. (1994). Medidas para asegurar la calidad en la educación superior. Revista de la Educación Superior, (96), 103-112. Recuperado de http://publicaciones.anuies.mx/pdfs/revista/Revista96_S3A2ES.pdf.

Fernández, A., Cristobal, J., Navarro, A., Fernández, A., Merino, J., Peralta, M. y Roldan, Y. (2010). El campus virtual de la Universidad Complutense de Madrid. Pixel-Bit. Revista de Medios y Educación, (32), 55-65. 
USO DE LAS TIC EN EL PROCESO DE ENSEÑANZA-APRENDIZAJE EN LOS PROFESORES UNIVERSITARIOS.

Fernández, E. (2017). Una mirada a los desafíos de la educación superior en México. Innovación educativa, 17(74), 183-207. Recuperado de http://www.scielo.org.mx/scielo.php?script=sci_arttext\&pid=S1665$26732017000200183 \&$ lng=es\&tlng=es.

García, L. (2017). Educación a distancia y virtual: calidad, disrupción, aprendizajes adaptativo y móvil. RIED. Revista Iberoamericana de Educación a Distancia, 20(2), 9-25. Recuperado de http://revistas.uned.es/index.php/ried/article/view/18737

Hernández, F., Maquilón, J., Monroy, F. e Izquierdo, T. (2010). Teacher perception of student learning needs according to the teachers' teaching area, gender and teaching approach. Documento presentado en el European Educational Research Association 2010, agosto. Helsinki, Finlandia.

Instituto Politécnico Nacional. (2004). Un nuevo modelo educativo para el IPN. México: IPN Instituto Politécnico Nacional. (2017). Informe de evaluación curricular UPIICSA: evaluación interna y externa del plan de estudios. México: IPN

Iarossi, G. (2006). The power of survey design: A user's guide for managing surveys interpreting results, and influencing respondents. Washington D.C., USA: World Bank.

Márquez, A. (2004). Calidad de la educación superior en México. ¿Es posible un sistema eficaz, eficiente y equitativo? Las políticas de financiamiento de la educación superior en los noventa. Revista Mexicana de Investigación Educativa, 9(21), 477-500.

McMillan, J. y Schumacher, S. (2011). Investigación educativa: una introducción conceptual $5^{\text {a }}$ ed. España: Pearson educación.

Merino, C. y Lautenschlager, G. (2003). Comparación estadística de la confiabilidad alfa de Cronbach: aplicaciones en la medición educacional y psicológica. Revista de Psicología de la Universidad de Chile, 12(2), 127-136. Recuperado de https://revistas.uchile.cl/index.php/RDP/article/download/17668/18430/.

Organización de las Naciones Unidas para la Educación, la Ciencia y la Cultura. (2009). Conferencia mundial sobre la educación superior: la nueva dinámica de la educación superior y la investigación para el cambio social y el desarrollo. París, Francia: UNESCO 
USO DE LAS TIC EN EL PROCESO DE ENSEÑANZA-APRENDIZAJE EN LOS PROFESORES UNIVERSITARIOS.

Richards, J. y Rodgers, T. (1992). Approaches and methods in language teaching: a description and analysis. Reino Unido: Cambridge University Press

Rivera, B. (2008). La "docencia virtual" como instrumento para la adaptación al EEES y herramienta de innovación docente: uso de "Blended Learning" en las clases de psicopedagogía y didáctica de la ERE en la Universidad de Jaén. Revista Iniciación a la Investigación.

Recuperado

de https://revistaselectronicas.ujaen.es/index.php/ininv/article/view/229

Rodríguez, H. (2014). Ambientes de aprendizaje. Ciencia Huasteca, 2(4). Recuperado de https://www.uaeh.edu.mx/scige/boletin/huejutla/n4/e1.html. 